\title{
SECADO DEL AJI PANCA EN UN SECADOR SOLAR FOTOVOLTAICO DE TUBOS AL VACIO
}

\section{DRYING AJI PANCA IN A SOLAR PHOTOVOLTAIC DRYER OF HEAT PIPE}

\author{
Juan Palo-Tejada ${ }^{1^{*}(\mathbb{D})}$ Alicia Puma-Taco ${ }^{1}$ [D Enriqueta Campos-Falcón ${ }^{1}$
}

${ }^{1}$ Facultad de Ciencias Naturales y Formales, Universidad Nacional de San Agustín de Arequipa, Perú

Recibido (Recieved): 21/02/2020 Aceptado (Accepted): 05/03/2020

\section{RESUMEN}

En este trabajo se realizaron pruebas del secado del ají panca en un secador solar de aire por coveccion forzada que utiliza quince tubos de vacío (Heat pipe), una cabina cerrada donde se coloca el producto y una turbina tipo jaula de ardilla que es alimentada por un sistema de dos paneles fotovoltaicos de 50W cada uno. El aire calentado es impulsado por la turbina desde los tubos al vacío hasta el producto que se encuentra en la cabina, en este trabajo se utilizó la ecuacion de difusión de Fick unidimencional y metodos númericos para determinar el coeficiente de transferencia de masa $2.61 \times 10^{-9} \mathrm{~m} / \mathrm{s}$ y el coeficiente de difusividad efectiva $1.52 \times 10^{-10} \mathrm{~m}^{2} / \mathrm{s}$ y se concluyo que la temperatura de secado de $55^{\circ} \mathrm{C}$ es la que mejor que se ajusta a los resultados estimados por el modelo. El secador solar de tubos al vacío es capaz de mantener una temperatura de operación entre $50^{\circ} \mathrm{C}$ a $55^{\circ} \mathrm{C}$ para una irradiancia de 450 a $1100 \mathrm{~W} / \mathrm{m}^{2}$ entre las 9:30 y las 14:30 horas, en estas condiciones, en dos días se consigue secar el aji panca hasta un $8 \%$ de contenido de humedad. El secado tradicional de este producto, toma entre siete y diez días.

Palabras Clave: secador solar de tubos de vacío, ají panca, sistema fotovoltaico.

\section{ABSTRACT}

In this work, tests were carried out on the drying of the ají panca in a forced air solar dryer using fifteen vacuum tubes (Heat pipe), a closed cabin where the product is placed and a squirrel cage turbine that is fed by a system of two photovoltaic panels of $50 \mathrm{~W}$ each. The heated air is driven by the turbine from the vacuum tubes to the product in the cabin, in this work the unidimensional Fick difusion equation and numerical methods were used to determine the mass transfer coefficient $2.61 \times 10^{-9}$ $\mathrm{ms}^{-1}$ and the effective diffusivity coefficient $1.52 \times 10^{-10} \mathrm{~m}^{2} \mathrm{~s}^{-1}$ and it was concluded that the drying temperature of is $55{ }^{\circ} \mathrm{C}$ is the one that best fits the results estimated by the model. The solar vacuum tube dryer is capable of maintaining an operating temperature between $50{ }^{\circ} \mathrm{C}$ to $55^{\circ} \mathrm{C}$ for an irradiance of 450 to $1100 \mathrm{~W} / \mathrm{m}^{2}$ between 9:30 am and 2:30 pm, under these conditions, in two days it is possible to dry the ají panca up to $8 \%$ moisture content. Traditional drying of this product takes between seven and ten days

Keywords: solar vacuum tube dryer, chili pepper, photovoltaic system.

\footnotetext{
* Corresponding author:

e-mail: jpalot@gmail.com
} 


\section{INTRODUCCIÓN}

El género Capsicum está compuesto por varias especies y una de ellas Capsicum Annuum L conocido en el medio local como ají panca, que se utilizan ampliamente para la nutrición humana y el procesamiento industrial debido a su notable color, aroma y acción fisiológica [8]. En el Perú el gobierno promulgó en el diario El PERUANO la Resolución Ministerial Nº434-2017-Minagri en la que se aprobó el "Plan de Desarrollo Sostenible de las especies del género Capsicum 2018-2028 [7]. Los diferentes métodos de procesamiento pueden alterar la calidad de los productos al mejorar o reducir la producción de compuestos bioactivos, uno de los principales métodos de procesamiento en el ají es el secado o deshidratado. El ají panca (Capsicum Annuum L) es usado en la preparación de alimentos en los centros de abastecimientos arequipeños se le encuentra en forma deshidratada. Los medianos y pequeños agricultores arequipeños deshidratan el ají exponiendo el producto directamente al sol, donde las condiciones ambientales como la humedad, polvo, smog, etc. contaminan el producto. Como consecuencia de este proceso se obtiene un producto de baja calidad.

\section{ANTECEDENTES}

Existe una gran variedad de ají que son usados para dar color o como condimento, por tal razón se han hecho estudios de secado del ají, por ejemplo en Hernandez [4] se estudió el secado de capsicum annuum $\mathrm{L}$ a través del modelo de Luikov, donde se analiza la influencia que tienen los diferentes números adimensionales en la perdida de humedad del chile jalapeño. En Ahmad [1] estudió el secado solar para el chile rojo; el producto se deshidrato hasta $10 \%$ de contenido de humedad en el secador solar en 33 horas de irradiancia. En Hernández [5] se estudió el secado del chile jalapeño; comparando los resultados obtenidos en el secador solar con los resultados del modelo desarrollado en base a la solución de Luikov. Tambien en Guzmán [3] se diseñó, desarrolló y controló un laboratorio portátil utilizando una filosofía de diseño mecatrónico para facilitar el estudio del proceso de secado de chile. Este laboratorio portátil puede simular diferentes condiciones de secado en un entorno controlado para encontrar los mejores parámetros de secado para diferentes tipos de chiles.

Convencionalmente las empresas de procesamiento de alimentos secan los productos usando combustibles no renovables, como petróleo, gas natural, o combustibles renovables como biomasa; pero hoy en día el uso de las energías renovables no contaminantes, como la energía solar, está siendo cada vez más considerada en diferentes áreas de la industria incluyendo el secado de alimentos, esto por la preocupación creciente en la contaminación del medio ambiente.

Para la realización de este estudio, se realizaron pruebas del secado del aji panca en el secador solar de tubos al vacio para calentar el aire y una turbina que impulsa el aire desde tubos al vacio hasta el producto, que se encuentra en la cabina; esta turbina tipo jaula de ardilla es alimentada por un sistema de dos paneles fotovoltaico cada uno de 50W. Los resultados experimentales registrados se compararon con los resultados estimados por modelo de difusion de Fick.

\section{METODOLOGÍA}

\subsection{EL SECADOR SOLAR}

El sistema empleado es un secador solar fotovoltaico de tubos al vacío Figura 1 para el secado de ají panca. Este secador utiliza 15 tubos al vacío de longitud $1.80 \mathrm{~m}$ y diámetro $0.095 \mathrm{~m}$ que se conectan al manifold; dentro de estos tubos se encuentran aletas de cobre y tubos de aluminio. Al manifold también se conecta una turbina de jaula de ardilla impulsada por un motor de corriente directa de $80 \mathrm{~W}$, la energía es suministrada por dos paneles fotovoltaicos, cada uno de $50 \mathrm{~W}$. Este sistema no posee acumuladores de energía, por lo tanto empieza a funcionar cuando la irradiancia es $400 \mathrm{~W} / \mathrm{m}^{2}$.

La turbina impulsa el aire caliente que se encuentra en los tubos al vacío y lo envía a la cabina donde se encuentra el ají panca. La cabina tiene dimensiones de $0.664 \times 0.503 \times 0.670 \mathrm{~m}$, dentro de esta se encuentra un soporte para cuatro bandejas, como se muestra en la figura 2, estas bandejas están unidas a un soporte que descansa sobre una balanza electrónica que mide la pérdida de masa del producto en tiempo real. En la cabina de secado se instalaron también sensores de humedad y temperatura.

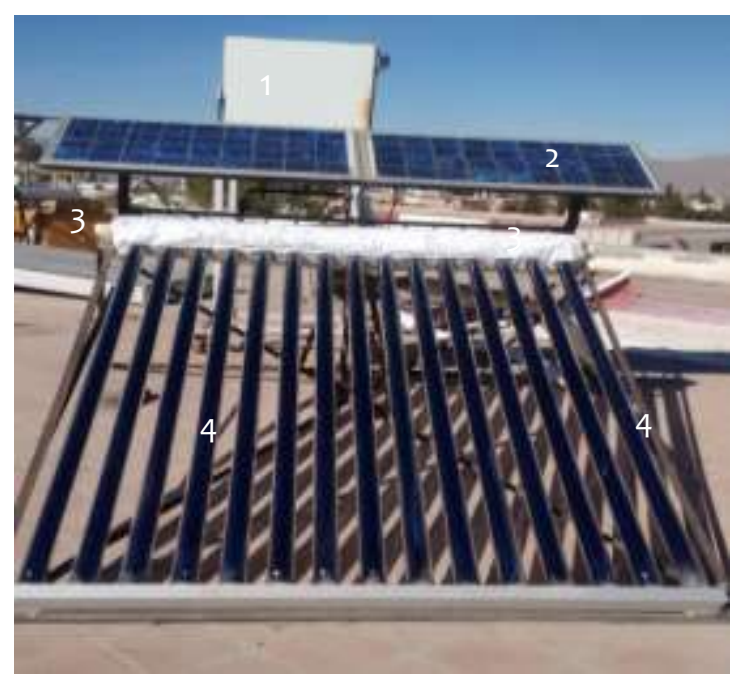

Fig. 1. Secador solar 
El secador solar está compuesto por 1: cabina de secado, 2: paneles fotovoltaicos, 3: manifold, 4: tubos al vacío.

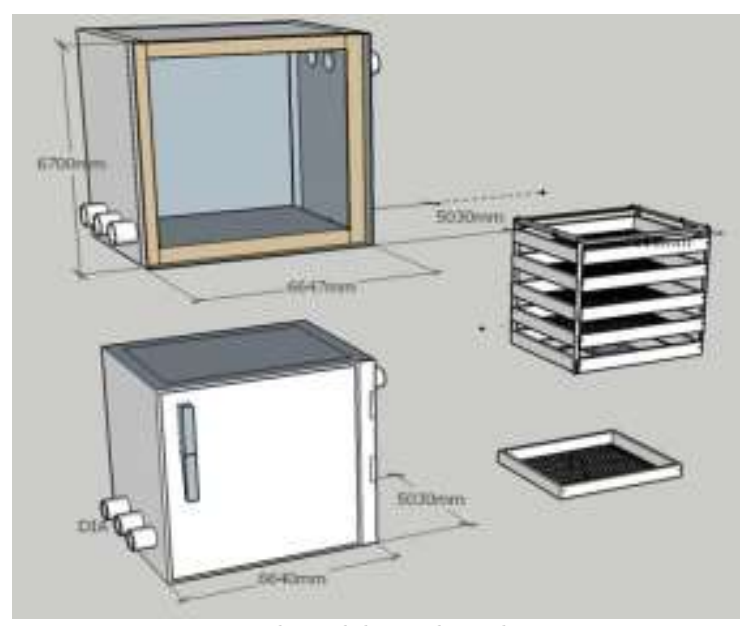

Fig. 2. Cabina del secador solar

La cabina para el secado solar tiene incorporado un soporte para cuatro bandejas.

\subsection{PREPARACIÓN Y SECADO DEL PRODUCTO}

Para el secado del ají panca primero se procedió a lavarlo, después se cortó longitudinalmente, extrayendo las semillas del producto, luego es colocado en las bandejas como se muestra en la figura 3 , estas bandejas son colocadas en el soporte que está dentro de la cabina. Aquí también se coloca el sensor que registrara la humedad y temperatura.

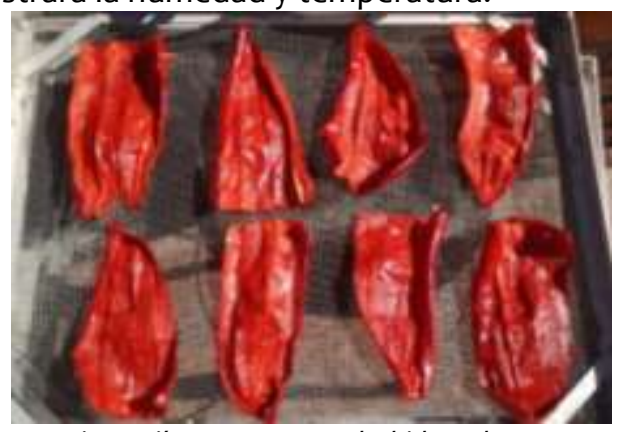

Fig. 1. Ají panca para ser deshidratado

\subsection{MODELO DE SECADO}

Consideremos el ají panca, como una lámina cuyo espesor es menor que sus otras dimensiones espaciales y que está ubicada en el origen de coordenadas. La Ecuación de difusión de Fick unidimensional [2] en este condiciones puede escribirse como

$$
\frac{\partial M}{\partial t}=D \frac{\partial^{2} M}{\partial x^{2}}
$$

Donde $D$ es el coeficiente de difusividad, $x$ el espesor de la piel del ají, $M$ es la humedad en función de la profundidad de la pulpa y del tiempo. Consideremos en $t=0$ la humedad igual a la humedad inicial $M_{o}$, como se muestra en la ecuación (2).

Condiciones Iniciales ( $\mathrm{Cl})$ :

$$
M_{(x, t=0)}=M_{o}
$$

Condiciones de Frontera (CF) en $x=0$ y $x=l$

$$
\begin{gathered}
\left.D \frac{\partial M}{\partial x}\right|_{x=0}=0 \\
-\left.D \frac{\partial M}{\partial x}\right|_{x=l}=h_{m}\left(M_{l}-M_{e q}\right)
\end{gathered}
$$

La solución de esta ecuación diferencial es:

$$
\begin{gathered}
\frac{M(x, t)-M_{e q}}{M_{o}-M_{e q}}= \\
\sum_{n=1}^{\infty} \frac{2 \operatorname{sen} \mu_{n}}{\mu_{n}+\operatorname{sen} \mu_{n} \cos \mu_{n}} \cos \left(\mu_{n} \frac{x}{l}\right) \exp \left(-\mu_{n}^{2} \frac{D . t}{l^{2}}\right)
\end{gathered}
$$

Tomando al primer término de la serie:

$$
\begin{gathered}
\frac{M(x, t)-M_{e q}}{M_{o}-M_{e q}}= \\
\frac{2 \operatorname{sen} \mu}{\mu+\operatorname{sen} \mu \cos \mu} \cos \left(\mu \frac{x}{l}\right) \exp \left(-\mu^{2} \frac{D . t}{l^{2}}\right)
\end{gathered}
$$

Aplicamos logaritmo en ambos miembros de la ecuación (6) tenemos:

$$
\begin{aligned}
& \ln \left(\frac{M(x, t)-M_{e q}}{M_{o}-M_{e q}}\right) \\
= & \ln \left(\frac{2 \operatorname{sen} \mu}{\mu+\operatorname{sen} \mu \cos \mu} \cos \left(\mu \frac{x}{l}\right)\right)-\left(\mu^{2} \frac{D \cdot t}{l^{2}}\right)
\end{aligned}
$$

La ecuación (6) tiene forma de $y=a-b \cdot x$, por lo tanto identificamos

$$
\begin{gathered}
a=\ln \left(\frac{2 \operatorname{sen} \mu}{\mu+\operatorname{sen} \mu \cos \mu} \cos \left(\mu \frac{x}{l}\right)\right) \\
b=\mu^{2} \frac{D}{l^{2}}
\end{gathered}
$$

\section{ANÁLISIS DE RESULTADOS}

Los resultados del cálculo de los coeficientes de difusividad efectivo $D$ y convectivo de transferencia de masa $h_{m}$ del secado del ají panca a temperatura constante, se muestra en la tabla 1 y tabla 2.

TABLA I

Parámetros de secado 


\begin{tabular}{ccc}
\hline Temperatura & $\boldsymbol{D}\left(\mathrm{m}^{2} / \mathbf{s}\right)$ & $\boldsymbol{h}_{\boldsymbol{m}}(\mathrm{m} / \mathrm{s})$ \\
\hline $\mathbf{4 0}{ }^{\circ} \mathbf{C}$ & $2,25 \times 10^{-10}$ & $2,51 \times 10^{-9}$ \\
$\mathbf{4 5}^{\circ} \mathbf{C}$ & $1,57 \times 10^{-10}$ & $2,11 \times 10^{-9}$ \\
$\mathbf{5 5}^{\circ} \mathbf{C}$ & $1,52 \times 10^{-10}$ & $2,61 \times 10^{-9}$
\end{tabular}

Nota: muestra los coeficientes de difusividad efectivo $D$ y convectivo de transferencia de masa $\mathrm{hm}$ para 3 temperaturas de secado, si trabajamos a $45^{\circ} \mathrm{C}$ los coeficientes que deberíamos remplazar en el modelo (ecuación 5) son $\mathrm{D}=1,57 \times \llbracket 10 \rrbracket^{\wedge}(-10) \quad\left(\mathrm{m}^{\wedge} 2 / \mathrm{s}\right)$ y $\mathrm{h} \_\mathrm{m}=2,11 \mathrm{x}$ $\llbracket 10 \rrbracket^{\wedge}(-9)(\mathrm{m} / \mathrm{s})$.

TABLA II

Estadísticos

\begin{tabular}{cccc}
\hline $\mathbf{N}^{\circ}$ datos & RMSE & MAE & $\mathbf{R}^{2}$ \\
\hline 36 & 0.0258 & 0.0212 & 0.9963 \\
43 & 0.0229 & 0.0191 & 0.9952 \\
37 & 0.0384 & 0.0306 & 0.9940 \\
\hline
\end{tabular}

Nota: muestra los estadísticos que nos permiten indicar que también aproxima el modelo a la curva de secado experimental, la figura 7 muestra visualmente esta aproximación.

Se ha registrado datos de temperatura y humedad dentro de la cabina, y la irradiación solar. Las cuales se muestran en la figura 4 y 5 .

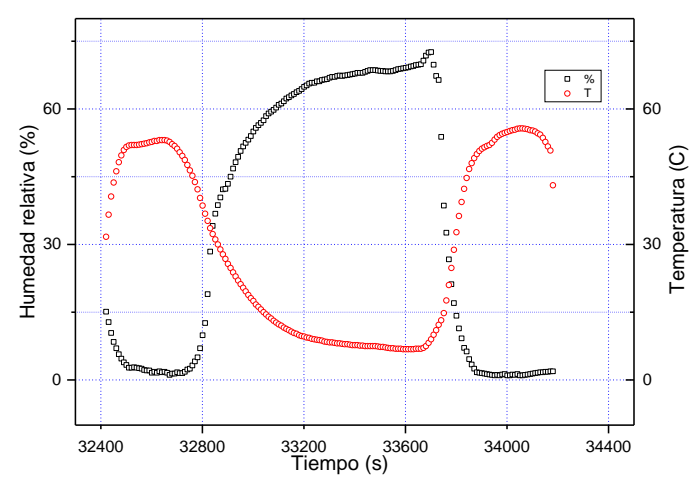

Fig. 2. Gráfica de temperatura y humedad dentro de la cabina

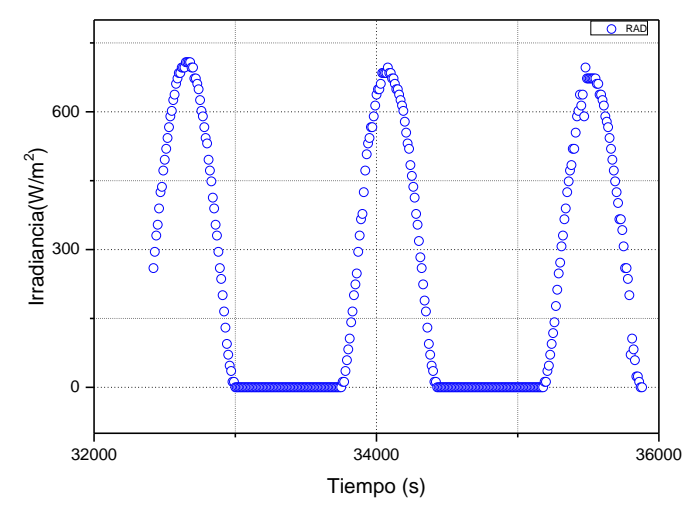

Fig.3. Gráfica de irradiancia
Con los datos registrados de masa y tiempo en el secador solar, se calculó la humedad del ají panca en base seca, con la ecuación siguiente:

$$
M=\frac{m-m_{s}}{m_{s}}
$$

Donde m: masa del ají panca y $m_{s}$ : masa seca del ají panca. Los resultados se muestran en la (Fig 6).

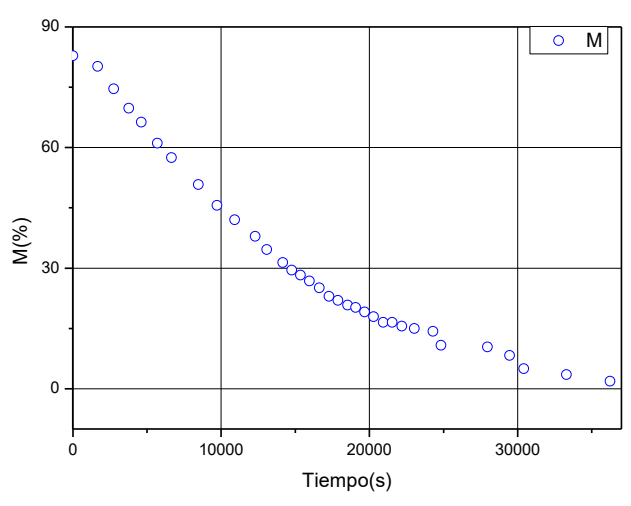

Fig. 4. Gráfica de la Humedad en base seca

Para comparar los resultados experimentales con los estimados por el modelo, graficamos $\frac{M-M_{e q}}{M_{0}-M_{e q}}$ en función del tiempo [6], Círculos en la figura 7 datos experimentales.

Utilizamos los parámetros del ajuste lineal, $a$ y $b$ para estimar los coeficientes $D$ y $\mu$ de las expresiones 7 y 8 , note que la ecuación (7) es trascendental por lo que utilizamos el procedimiento de Newton Raphson para encontrar sus raíces.

Utilizamos los coeficientes $D$ y $\mu$ para ajustar el modelo que se muestra en la ecuación (5), y graficamos el resultado en la figura 7 , línea continua.

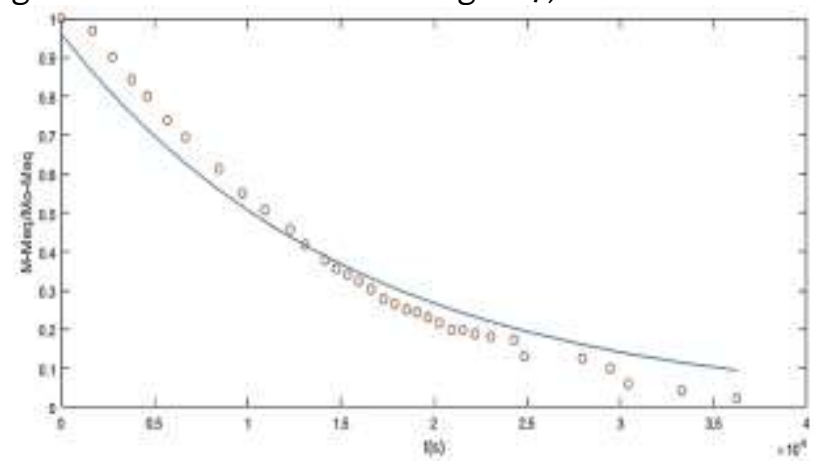

Fig. 5. Comparación de los resultados teóricos y la parte experimental

Se observa que el tiempo de secado del ají panca, en el secador de tubos al vacío de fue de 10.5 horas, en comparación con el secado tradicional que es de 7 a 10 días, en condiciones favorables.

De acuerdo con los ensayos realizados en un horno eléctrico y aplicado el modelo de difusión (teórico) la temperatura de secado óptimo para el ají panca es $55^{\circ} \mathrm{C}$. Los datos registrados en el secador solar 
fotovoltaico de tubos al vacío alcanzan temperaturas entre $50^{\circ} \mathrm{C}$ a $55^{\circ} \mathrm{C}$; por lo que este secador solar es óptimo para deshidratar el ají panca.

De esta información se obtuvo que la temperatura de operación del secador solar es de $50^{\circ} \mathrm{C}$ a $55{ }^{\circ} \mathrm{C}$, desde las 9:40 horas hasta las 14:00 horas; por lo que este secador solar es óptimo para deshidratar el ají panca.

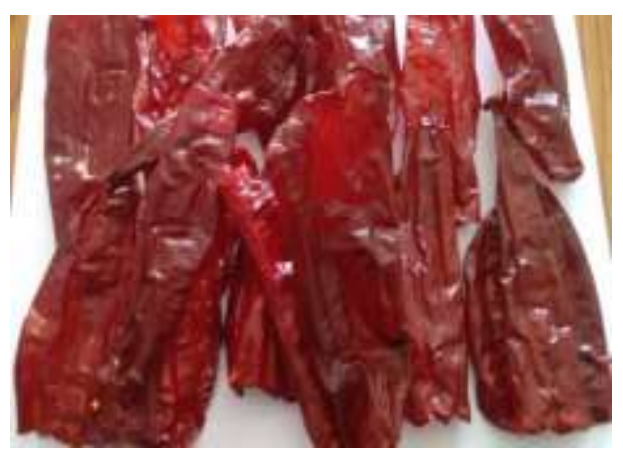

Fig. 6. Muestra del ají panca después de ser deshidratado

Con este modelo se puede predecir a que temperatura se debe secar el ají panca.

\section{CONCLUSIONES}

- En este trabajo se construyó y probó un secador solar de convección forzada de tubos al vacío.

- Se utilizó el secador solar para el secado del ají panca.

- Se calcularon los coeficientes de difusión y convectivo de transferencia de masa.

- Se comprobó que el modelo se ajusta mejor a los datos experimentales cuando la temperatura de secado es de $55^{\circ} \mathrm{C}$.

\section{AGRADECIMIENTOS}

Agradecemos a la Escuela profesional de Física de la Universidad Nacional de San Agustín de Arequipa, y al vicerrectorado de investigación por facilitarnos los equipos y laboratorio para realizar este trabajo

\section{REFERENCIAS}

[1] A. Fudholi, M. Y. Othman, M. H. Ruslan and K. Sopian, "Drying of malaysian capsicum annuum L. (Red Chili) dried by open and solar drying", International Journal of Photoenergy, vol. 2013, pp. 1-9, feb. 2013.

[2] F. J. Arranz, E. C. Correa, H. T. Jímenez, B. Diezma, J. GarcíaHierro, J. I. Robla and P. Barreiro, "Empleo de métodos numéricos para el ajuste de los coeficientes de difusividad (D) y convectivo de transferencia de masa ( $\mathrm{hm}$ ) en el secado de alimentos", in VI Congreso Ibérico de Agroingeniería, Portugal, 2011.

[3] C. H. Guzmán Valdivia, J. L. Carrera Escobedo, M. A García Ruíz, A. Ortíz Rivera and O. Désiga Orenday, "Design, development and control of a portable laboratory for the chili drying process study", Mechatronics, vol. 39, pp. 160-173, 2016.

[4] J. Hernández, P. Quinto, J. Cuevas, R. Acosta and J. Aguilar, "Estudio Del Secado De Capsicum Annuum L a Través Del Modelo De Luikov”, Caos Conciencia, vol. 1, pp. 21-30, 2008.
[5] J. Hernández, P. Quinto, J. Cuevas, R. Acosta and J. Aguilar, "Estudio Del Secado De Productos Agricolas", 2008.

[6] D. M. Kadam, R. K. Goyal, K. K. Singh and M. K. Gupta, "Thin layer convective drying of mint leaves", Journal of Medicinal Plants Research, vol. 5, no. 2, pp. 164-170, 2011.

[7] MINAGRI (2017, Oct 24). Plan de Desarrollo Sostenible de las especies del género Capsicum 2018-2028 [online]. Available: https://busquedas.elperuano.pe/normaslegales/aprueban-el-plan-dedesarrollo-sostenible-de-las-especies-d-resolucion-ministerial-n-04342017-minagri-1580151-1/

[8] K. Sanatombi and S. Rajkumari, "Effect of Processing on Quality of Pepper: A Review", Food Reviews International, pp. 1-18, 2019.

\section{(c) (1)}

Los artículos publicados por TECNIA pueden ser compartidos a través de la licencia Creative Commons: CC BY 4.0. Permisos lejos de este alcance pueden ser consultados a través del correo revistas@uni.edu.pe 